\title{
Finite element method for rock plate impact test
}

\author{
Hongwei Fan ${ }^{1, a}$, Le Hu ${ }^{2, b}$, Yuxun Tang ${ }^{3, c}$ and Guoqin Wang ${ }^{4, d}$ \\ 1,2,3,4 Zhejiang institute of furniture and hardware, Hangzhou, China, 310013 \\ ${ }^{1,3}$ Key Laboratory of Furniture Inspection Technology of Zhejiang Province \\ afanhw88@126.com, bhlcar001@icloud.com, 'cyxtom@sina.com, dnoahwang2009@sina.com
}

Keywords: Rock plate, Impact test, Finite element, Explicit dynamics

Abstract. Base on the test method in EN 12572-1-2007, a finite element model of rock plate impact test have been established. It can solve the maximum stress and strain of the rock plate and the impact test equipment, analysis the structural failure point. By changing impact height, it can be used to solve different influence on rock plate under different test condition. The results show that the maximum stress of rock plate is subjected to a nonlinear variation. All data obtained from the numerical simulation can provide reference to perfect the test method of national standard.

\section{Introduction}

According to 'The announcement of first batch of high risk sports project directory' and 'The management measures for license of high risk sports venues', published in May 2013, by General Administration of Sport, sports departments need to work on high risk sports venues to carry out the administrative licensing. In sport climbing place, for example, the product quality supervision work can be reference to the current national standard: GB 19079.4-2014 'Operation conditions and technical requirements for gymnasium and playground. Part 4:Sport climbing place'. The standard include the technical requirements of climbing line, the top protection system, protection point, static load of rock plate, dynamic load of rock plate and so on, but lack of test method. Dynamic load of rock plate, for example, if the rock plate installation conditions, the size of impact head, impact velocity or other factors have been changed, the test results will be affected. Therefore, the test results have no repeatability. The third-party testing agency unable to carry out. Administrative examination and approval work will also be impeded. So, studying and mastering the impact test technology of rock plate, perfecting test method in Chinese national standard have great significance.

At present, based on the development of finite element technology and Explicit dynamics technology, analyzing object collision through numerical simulation methods, have been gradually adopted by mechanical designer. In addition, the numerical simulation analysis can get intuitive, quantitative and accurate details of the stress, strain and displacement field information.

This study established a rock plate impact model, based on finite element technology; refer to the EN 12572-1-2007 'Artificial climbing structures. Part 1: Safety requirements and test methods for the ACS with protection, points', carried out numerical simulation and analyzed mechanical properties, to provide evidence for test methods and manufacturing test equipment.

\section{The impact test of rock plate}

The clause 4.5 of EN 12572-1-2007 'Artificial climbing structures. Part 1: Safety requirements and test methods for the ACS with protection points' show that after the test, there shall be no breaking or splitting of any surface element. The sample should be a standard element or a specially made flat panel $1 \mathrm{~m} \times 1 \mathrm{~m}$. A sample should be set up for simulating the actual installation conditions, is shown in Fig.1. A indenter will be released from $1500 \mathrm{~mm}$ in height, striking the geometry center of the rock plate, is shown in Fig.2. 


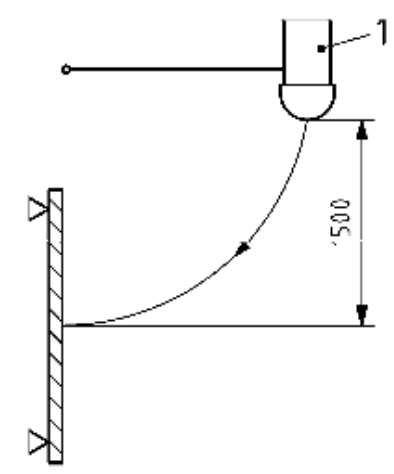

a) horizontal impact

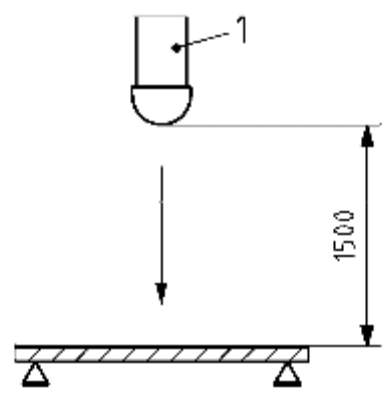

b)vertical impact

Key

1 indenter

Fig.1 Impact test

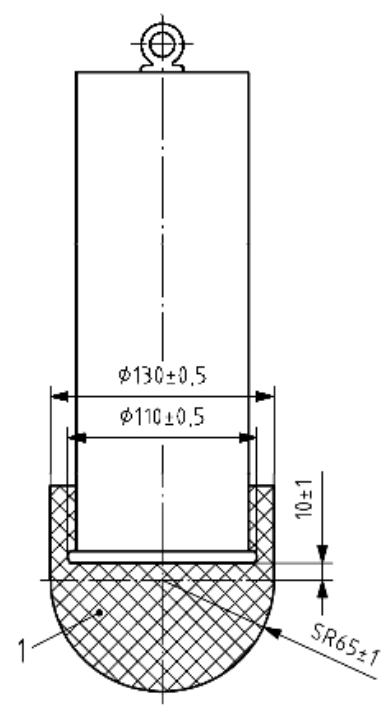

Fig.2 Indenter

\section{Numerical analysis model}

The model consists of rock plate and indenter, is shown in Fig.3. All the material parameters are set in Explicit Dynamics solver. This study selected three kinds of material in the material storage, CONC $35 \mathrm{Mpa}$, Rubber1, and Structural Steel, corresponding rock plate, buffer impact head and impact body. The size of the rock plate model is $1 \mathrm{~m} \times 1 \mathrm{~m} \times 0.05 \mathrm{~m}$. And the impact body's length is $0.35 \mathrm{~m}$. In meshing, ' Physics Preferences' is set to 'Explicit', 'Relevance' is set to '0', 'Size correlation' is set to 'Medium'. After meshing, there are 9518 units and 3539 nodes, is shown in Fig.4.

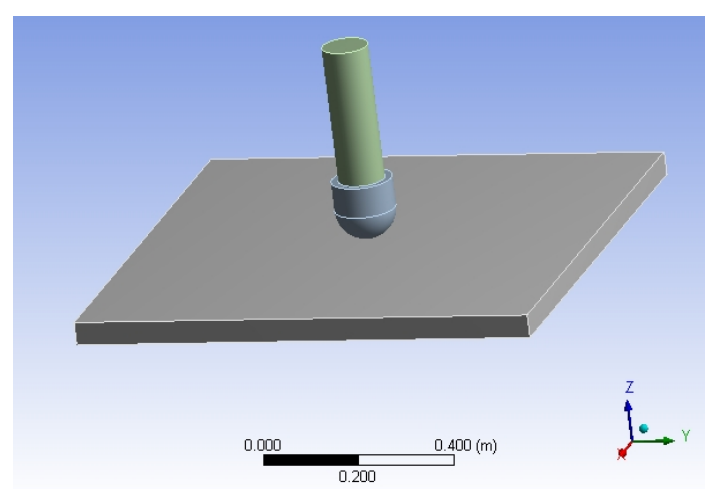

Fig.3 Model 


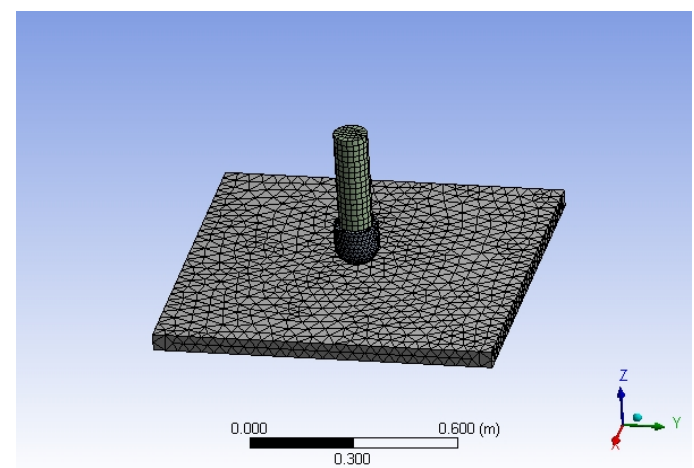

Fig.4 Meshing

\section{Numerical simulation and the result analysis}

According to test method in European standard, the impact height is $1.5 \mathrm{~m}$. According to the calculated formula of free fall, instant impact speed is $5.422 \mathrm{~m} \cdot \mathrm{s}^{-1},-\mathrm{z}$ direction. According to actual installation condition, the rock plate edges are added the 'Fixed Support' constraint. The end time is $0.01 \mathrm{~s}$.

After numerical simulation of impact test, the stress of rock plate and the impact head evenly spread around by the geometric center of the impact point, larger values appear in the center of the impact point and plate fixed place, is shown in Fig.5. The max value of stress is $2.208 \times 10^{6} \mathrm{~Pa}$ appearing the geometric center of the rock plat. After the impact, rock plate will collapse in - $\mathrm{z}$ direction. The stress value of back face is higher than positive. On four fixed surface, the near impact center, the higher the stress value. In the process of design, production and installation, we can consider to add reinforcement on the rock plate's back, enhancing the capacity of resistance to deformation. In the center of the rock plate edges, we can also consider to design fixed point, to strengthen the stability of shocks.

In test system, the peak stress value appeared on impact body, attaching to the buffer impact head, the value is $3.5203 \times 10^{6} \mathrm{~Pa}$. In the design of test equipment, we can consider to rounding off the bottom of the cylinder, reducing stress concentration. The stress concentration of buffer layer appears at the impact geometry center, value of $1.2499 \times 10^{6} \mathrm{~Pa}$. There is large deformation appeared in geometry center, maximize strain value is $1.1118 \mathrm{~m} / \mathrm{m}$.

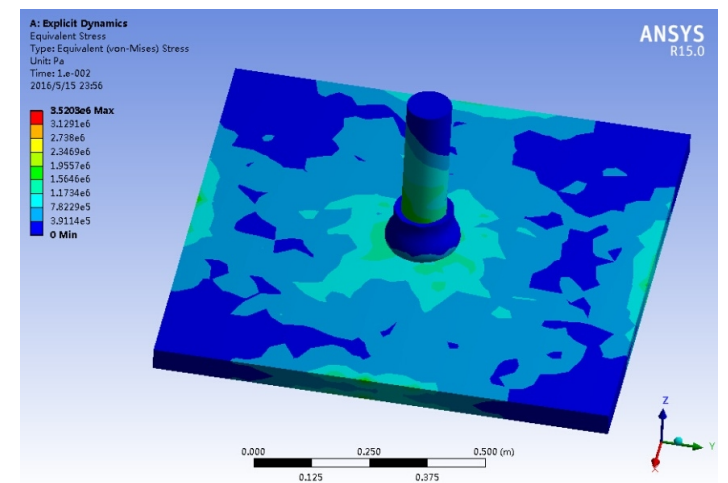

Fig.5 Equivalent stress nephogram

\section{Comparison and analysis of different impact height}

A series of parameters of stress of different impact height is calculated, is shown in Table 1. In each simulation, the maximum stress of rock plate are focused on the back of the impact face. Impact height were set to $1 \mathrm{~m}, 1.5 \mathrm{~m}, 2 \mathrm{~m}, 2.5 \mathrm{~m}$. As the height increase, the maximum stress of rock plate respectively increases $30.0 \%, 43.2 \%, 33.1 \%$. So, adjusting the impact height, impact test results will be affected. The maximum stress of impact head and buffer layer is also increased with the increase of 
impact height. Especially, when the impact height increased from $2 \mathrm{~m}$ to $2.5 \mathrm{~m}$, the maximum stress is increased $217 \%$. Test equipment is prone to failure and damage. It should be considered in design.

Table 1 Results of different impact height

\begin{tabular}{ccccc}
\hline \multirow{2}{*}{ Impact hight $(\mathrm{m})$} & $\begin{array}{c}\text { mpact velocity } \\
(\mathrm{m})\end{array}$ & Rock plate & Impact head & Buffer layer \\
\cline { 3 - 5 } & 4.427 & $1.8351 \times 10^{6}$ & $4.6619 \times 10^{6}$ & $0.9026 \times 10^{6}$ \\
\hline 1 & 5.422 & $2.3856 \times 10^{6}$ & $5.2859 \times 10^{6}$ & $1.2499 \times 10^{6}$ \\
1.5 & 6.261 & $3.4165 \times 10^{6}$ & $6.9317 \times 10^{6}$ & $1.5384 \times 10^{6}$ \\
2 & 7.000 & $4.5499 \times 10^{6}$ & $21.95 \times 10^{6}$ & $1.6813 \times 10^{6}$ \\
\hline
\end{tabular}

\section{Conclusions}

Based on finite element technology, rock plate impact test model was established. After the simulations of impact test under different heights, the conclusion is as follows:

(1) Through numerical simulation, the stress distribution of rock plate and the impact head can be calculated, and failure position can be got. It can provide a reference for the design of rock plate and test equipment.

(2) Through the results of numerical simulation of different height impact, the results showed that the linear increase of impact high will generate nonlinear growth of the maximum stress of rock plate.

(3) This study use the numerical simulation technology to analyze rock plate impact test. The result provide a reference to perfect the test method.

\section{Acknowledgements}

This work was financially supported by the science and technology plan projects of AQSIQ(20147QK207).

\section{References}

[1] Hao Tang, Junli Zhao, Kai Wang, Zhiqiang Wang: Mechanical \& Electrical Technology Vol. 2 (2015), p. 14

[2] Xiaolong Deng, Guosheng Feng, Pengfei Li: Journal of Chengde Petroleum College, Vol. 17 (2015), p. 39

[3] Shuyi Yang: Drop Impact Dynamics and Crashworthiness Robust Design for Manufactured, China University of Mining and Technology(2009)

[4] Yi-xian Wang, Ping Cao, Tu-bing Yin, Journal of Sichuan University(Engineering Science Edition), Vol. 43 No.6(2011),p. 85

[5] Gui-min Zhang,Xiu Yu, Yan-hong Ma,Xiang-yan Chen, Zhi-qiang Jiang, China Sport Science, Vol. 31 No.8(2011.),p.20-26

[6] Information on http://www.sport.gov.cn/ 